\title{
Références bibliographiques du dossier « L'école en milieu rural »
}

\section{(2) OpenEdition \\ Journals}

Édition électronique

URL : http://journals.openedition.org/ries/3334

DOI : $10.4000 /$ ries.3334

ISSN : 2261-4265

Éditeur

Centre international d'études pédagogiques

\section{Édition imprimée}

Date de publication : 1 juin 1996

Pagination : 133-138

ISSN : $1254-4590$

Référence électronique

"Références bibliographiques du dossier «L'école en milieu rural » », Revue internationale d'éducation de Sèvres [En ligne], 10 | 1996, mis en ligne le 30 juillet 2013, consulté le 19 avril 2019. URL : http:// journals.openedition.org/ries/3334; DOI : 10.4000/ries.3334

Ce document a été généré automatiquement le 19 avril 2019

(c) Tous droits réservés 


\section{Références bibliographiques du dossier «L'école en milieu rural »}

1 Cette bibliographie thématique présente une sélection de documents récents, pour la plupart consultables au CIEP. Elle est organisée, à partir de références françaises et étrangères, en fonction des deux grandes orientations du numéro :

- L'école rurale dans le monde scolaire : quelle pédagogie ? quelle organisation?

- L'école dans le monde rural : quelle place dans l'aménagement du territoire? Quelle école pour quel développement?

\section{École rurale et système éducatif}

\section{Pédagogie et scolarité à l'école rurale}

2 DEYOUNG Alan J. (ed.), "Rural Schools", Phi Delta Kappan, novembre 1995, vol. 77, n², p. 104-148.

Les articles de ce dossier présentent différents aspects de l'enseignement rural aux ÉtatsUnis : composantes de l'éducation, degré d'introduction des innovations technologiques, prise en compte des spécificités, objectifs et aspects de la réforme de l'école rurale.

3 "L'école rurale à l'avant-garde ? ", L'Éducation enfantine, sept. 1991, $n^{\circ}$ hors-série, p. 30-35.

Menée dans les départements pilotes, une enquête sur la mise en place des cycles à l'école montre différents visages de cette réforme. En milieu rural, certaines orientations sont déjà entrées dans les faits, qu'il s'agisse de gérer l'hétérogénéité, d'assurer le suivi des élèves ou de décloisonner.

4 "École rurale, communication et technologies nouvelles", actes du colloque international d'Autrans, juillet 1995, Longechenal, CREPSC, 1995, 275 p.

L'école rurale est un terrain privilégié d'investigation en ce qui concerne l'intégration des technologies de l'information et de la communication dans le système éducatif, comme le montre la réussite d'un certain nombre d'expériences. Les apports des nombreux intervenants, venus d'horizons professionnels et géographiques variés, fournissent des perspectives intéressant l'ensemble de la communauté éducative. 
FERMANEL Didier, Gérer une classe unique, Evreux, CDDP de l'Eure, 1994, cassette vidéo : 22 minutes.

Les images fournissent exemples et références pour mieux gérer le temps et l'espace. Quatre domaines sensibles sont abordés : autonomie des élèves, maitrise des groupesateliers, mode de structuration des connaissances, gestion de la section maternelle.

GROS André, METZGER André, «Le collège, structure de scolarisation en milieu rural », Cahiers Binet-Simon, 1991, n० 626-627, p. 95-111.

À l'intérieur d'un numéro qui traite de «l'éducation rurale » sous différents aspects, cet article montre les transformations induites par la création des collèges ruraux, tant sur le cursus scolaire des enfants que sur les comportements des équipes éducatives face aux nouvelles attentes des élèves et des parents. Il se base pour cela sur l'expérience vécue par les auteurs dans un collège de l'Aisne.

LEROY-AUDOUIN Christine, MINGAT Alain, "Les groupements d'élèves dans l'école primaire rurale en France: efficacité pédagogique et intégration des élèves au collège", Les Notes de l'IREDU, 1996, $n^{\circ} 1$, p. 1-4.

Trois enquêtes ont été réalisées auprès d'échantillons représentatifs d'élèves de Saône-etLoire et de l'Yonne, scolarisés dans des classes à cours simple ou à cours multiples. Elles permettent d'analyser les relations entre ces modalités de scolarisation et les acquisitions scolaires et carrières des élèves.

PLÖSSNING Franz, SCHRATZ Michaèl, "The Rural Secondary School: an Austrlan Reform Project for School Autonomy", International Review of Education = Revue internationale de pédagogie, 1991, vol. XXXVII, $n^{\circ}$ 2, p. 267-275.

Cette communication fait le point sur les différents aspects du projet de réforme de l'enseignement secondaire rural en Autriche : objectifs, éléments, contrôle, évaluation.

9 THIRIOT Josiane, DENAVARRE Catherine, ROSENBERG Fabrice, "Une école, des écoles", JDI (Journal des instituteurs et des institutrices), oct. 1990, p. 49-63.

Ce dossier illustre la diversité des écoles primaires. En ce qui concerne le milieu rural, les différents reportages (écoles à classe unique, ramassage scolaire, opinion des parents, activités extra-scolaires) présentent des situations concrètes.

\section{Organisation de l'école rurale}

"Agricultural and Rural Education", in International Encyclopedia of Education, Oxford, Pergamon, 1994, 1.1, p. 240-244.

Cet article de l'encyclopédie fournit un aperçu sur les aspects spécifiques de l'enseignement rural : contraintes de taille et d'isolement, éducation liée à un mode de vie.

11 CAVICCHIONI Vittoria, ERIKSON Anna, Urban and Rural Schools: Spécial Survey on Primary Education, Paris, Unesco, déc. 1992, 17 p., (Statistical issues).

Les écoles rurales sont souvent moins complètes que les écoles urbaines, notamment dans les pays en développement et particulièrement en Afrique, et leur capacité est toujours moins élevée, aussi bien en ce qui concerne le nombre d'élèves accueillis que le nombre de classes. Par contre, par rapport à la population scolaire, les écoles rurales sont généralement plus nombreuses que les écoles urbaines.

ET DE L'INSERTION PROFESSIONNELLE, «Les zones d'habitat dispersé: un handicap pour les 
études? » in Rapport 1995 Inspection générale de l'administration de l'Éducation nationale, Paris, la Documentation française, 1995, p. 55-100.

L'habitat dispersé est une notion mal définie, inégalement prise en compte et difficilement traitée. Les données nationales montrent de fortes disparités géographiques, des évolutions fluctuantes et des efforts, notables mais inégaux, d'adaptation du réseau scolaire. Face à la diversité des situations, des inégalités subsistent et la gestion est parfois approximative. Les résultats du service éducatif présentent des aspects positifs, mais des évolutions sont nécessaires.

CEUVRARD Françoise (réd.), » Le système éducatif en milieu rural », Éducation et formations, oct.1995, $n^{\circ} 43$, p. 5-155.

En rassemblant et mettant en forme les données statistiques disponibles, ce numéro apporte des éléments de réflexion au débat français actuel. L'alternative entre service public de proximité et qualité de l'enseignement par le regroupement des structures scolaires doit être nuancée. Pour ce faire, de nombreuses informations sont apportées sur: la localisation et la taille des établissements; les coûts, ressources et moyens; les enseignants ; les conditions de vie, acquis et cursus des élèves.

ORGANISATION DE COOPÉRATION ET DE DÉVELOPPEMENT ÉCONOMIQUES, «L'infrastructure de l'éducation dans les zones rurales ", Les cahiers du PEB, Pans, OCDE, 1994, 36 p.

Faisant suite au séminaire de 1993 sur ce thème, ce rapport présente une synthèse des travaux du « Programme pour la construction et l'équipement de l'éducation » de l'OCDE. Modes d'organisation du réseau scolaire, fonctions de l'école rurale, considérations financières et conception des écoles en milieu rural sont les différentes approches envisagées.

ORGANISATION DE COOPÉRATION ET DE DÉVELOPPEMENT ÉCONOMIQUES, «L'éducation en zone rurale ", PEB Échanges, févr. 1994, n 21, p. 1-17.

En complément au rapport, ce numéro présente quelques-uns des thèmes traités, ainsi que des résumés d'études de cas, qui donnent un aperçu de la variété des situations.

PISKUNOV Sergei Antonovich, Education in the Countryside and Teachers' Training for the Rural School, Orel (Russie): Pedagogical Institute, juillet 1992, $7 \mathrm{p}$.

Texte d'une communication faite par le recteur de l'Institut pédagogique d'Orel, région située au sud-ouest de Moscou, sur l'éducation en zone rurale et la formation des enseignants qui y travaillent.

PSACHAROPOULOS George, ROJAS Carlos, VELEZ Eduardo, «Achievement Evaluation of Colombia's "Escuela nueva": is Multigrade the Answer?", Comparative Education Review, août 1993, vol. XXXVII, $n^{\circ}$ 3, p. 263-276.

Les auteurs font un bilan du programme qui visait à développer, en zone rurale, des écoles offrant l'ensemble des cinq années de l'enseignement primaire dans une classe à plusieurs niveaux. Les résultats sont positifs, comme le montrent les évaluations statistiques de la phase expérimentale, mais la généralisation de l'expérience pose des problèmes.

18 TORRES Rosa Maria, "Nouvelles formules d'enseignement scolaire: le programme colombien "Escuela nueva" ", Perspectives, 1992, vol. XXII, $n^{\circ} 4$ (84), p. 573-584.

Ce programme, à l'origine expérience locale, est devenu une ligne politique nationale, en particulier pour l'enseignement primaire en milieu rural. Il s'intègre dans le cadre du système scolaire et voit son influence s'étendre au-delà des frontières. L'auteur nous donne ses caractéristiques essentielles, en analyse les points positifs mais aussi les 
faiblesses. Le modèle est-il exportable dans la mouvance de la stratégie mondiale de l'éducation pour tous?

\section{École et monde rural}

\section{École rurale et aménagement du territoire}

19 BOUCHAT Thérèse-Marie, Les voix de l'école rurale, Montreuil, Vie ouvrière, 1992, 147 p., (Coll. EVO-Société).

Cet ouvrage regroupe des témoignages de parents, enfants, enseignants, qui racontent l'école rurale en Belgique telle qu'ils la voient et la vivent.

20 "L'école des maires; la communale an 2000 », Le Monde de l'éducation, juin 1995, n 227, p. 14-26. La question scolaire est un des enjeux importants des élections municipales, comme le montre l'enquête menée sur le terrain à travers les régions françaises. Politique locale de l'enseignement, place de l'école rurale, rôle du partenariat, cumul des fonctions d'enseignant et de maire ou de secrétaire de mairie, tels sont les différents points abordés dans les articles de ce dossier.

21 "L'école en milieu rural : dossier ", Animation et éducation, n 115, juillet-août 1993, p. 7-28.

Dans le débat actuel, les points de vue sont divers, mais tous s'accordent sur la nécessité d'une école «nouvelle» favorisant le maintien d'un monde rural vivant et dynamique. Les témoignages d'enseignants montrent que l'école et la coopération ont un rôle important à jouer dans cette dynamique socio-économique.

GARDOU Charles (coord.), «L'éducation rurale », Cahiers Binet-Simon, 1991, n 626-627, p. 1-191.

Ce numéro présente une réflexion sur la part que peut prendre l'éducation dans le devenir du monde rural et sur le lieu de créativité extra- et post-scolaire qu'il a représenté. Les différents articles portent sur l'histoire et la sociologie du monde rural, puis sur la notion même de rural et l'avenir de l'agriculture, enfin sur les réponses apportées par les institutions scolaires ou extra-scolaires et la conception d'une formation initiale et continue adaptée.

23 "Lire et écrire en zone rurale ", actes du Forum Éducation nationale-Culture, 18 mai 1994, SaintJust-en-Chaussée, Amiens, CRDP, 1994, $110 \mathrm{p}$.

Les intervenants s'interrogent sur la place et le rôle de l'espace rural en France et plus particulièrement en Picardie. Ils montrent la spécificité du système éducatif dans ce milieu et présentent les actions qui y sont menées en faveur de la lecture et de l'écriture.

MABIT René, MINGAT Alain, OGIER Cédric, "L'école rurale: Du partenariat au développement coopératif. Éléments pour une réflexion nouvelle sur l'école primaire en milieu rural », Savoir : éducation, formation, janv.-mars 1994, $n^{\circ} 1$.

Le premier texte indique pour quelles raisons la conception du développement doit évoluer et comment cela peut se faire, en particulier en réalisant "un aménagement éducatif du territoire au service de la qualité de l'éducation ». Le second article présente, à partir d'une simulation concernant cinq cantons ruraux de la Nièvre, les différentes possibilités d'organisation de la carte scolaire et leurs conséquences pédagogiques et budgétaires.

MAUGER Pierre (dir)., Agir ensemble pour l'école rurale, Paris, ministère de l'Éducation nationale et de la Culture, direction de l'Information et de la Communication, 1992, $145 \mathrm{p}$.

Après une courte présentation du déclin démographique du milieu rural, ce document 
s'intéresse aux solutions apportées sur le plan de l'enseignement, essentiellement par les regroupements pédagogiques intercommunaux. Plusieurs réalisations concrètes sont présentées. Le dernier chapitre s'interroge sur les formes que peut prendre l'école rurale pour favoriser à la fois l'aménagement du territoire et l'éducation des enfants.

MINISTÈRE DE L'ÉDUCATION NATIONALE, «Le réseau scolaire en milieu rural » in Rapport de l'Inspection générale de l'Éducation nationale, juin 1995, Paris, la Documentation française, 1995, p. 543-582.

Un chapitre de ce document annuel est consacré cette année au travail effectué par la commission «Zones rurales ». Conditions actuelles de scolarisation, objectifs du système, rôle des différents partenaires dans le cadre d'une responsabilité partagée, propositions, tels sont les différents volets de cette synthèse.

NOIROT Paul (coord.), « Rural : une carte pour la France », Panoramiques, $n^{\circ}$ 18, 1995, p. 1-188.

Ce numéro thématique analyse la problématique du monde rural français selon quatre axes: nouvelles fonctions de l'espace, agriculture, développement local, culture. A l'intérieur de cette dernière partie, l'article d'Yves Jean est consacré à l'école rurale.

\section{École rurale et développement}

29 ACIKGOZ Kemal, «Rural Development by Teacher Training: the Village Institutes in Turkey, Fifty Years ago", Zeitschrift fur internationale Erziehungs- und socialwissenschaftliche Forschung, 1995, $n^{\circ} 1, p .91-102$.

Les « instituts de village », qui ont fonctionné en Turquie de 1940 à 1950, avaient pour objectif la formation des enseignants des écoles primaires rurales et, à travers elle, la transformation des zones rurales. Malgré leur brève existence, ils ont eu un impact important sur le développement de l'éducation rurale et, par elle, sur l'évolution du monde rural.

CLEVERLEY John, "On the Evidence before Me..." Putting the Case for Rural Educational Reform in China ", Comparative Education, 1991, vol XXVII, $n^{\circ} 1$, p. 53-60.

Universitaire australien spécialiste de l'enseignement en Chine, l'auteur présente la réforme de l'enseignement rural dans ce pays où $80 \%$ de la population vit en dehors des grandes villes. Il cherche à montrer les grandes orientations, politiques, idéologiques et historiques, liées au contexte et au contenu du débat sur l'éducation.

31 "Developing Rural Schools, a Key to Community Growth: Lectures, Papers, Documents", Interskola Conference, Boda, Norway, 24-31 July 1992, Norvège, Nordland County Director of Education, $343 \mathrm{p}$.

Actes du colloque inter-scolaire sur «Le développement des écoles rurales, clé de la croissance communautaire ", qui rassemblait des participants norvégiens, australiens, écossais et allemands.

32 EKANAYAKE S. B., "La pédagogie en zone rurale: comment promouvoir le développement rural à la base ", Perspectives, 1990, vol. XX, $n^{\circ} 1$, p. 129-143.

Dans les pays en développement, à prédominance rurale, les systèmes d'enseignement adoptés sont parfois inadaptés aux besoins de la population et à l'environnement socioculturel. L'auteur décrit le schéma d'un plan d'action pour une pédagogie rurale et présente, pour illustrer son propos, le cas du Sri Lanka.

GORMAN Kathleen S., POLLITT Ernesto, "School Efficiency in Rural Guatemala ", International

Review of Education = Revue internationale de pédagogie, sept. 1992, vol. XXXVIII, $n^{\circ} 5$, 
p. 519-534.

Destinée à évaluer le rendement scolaire à partir d'enquêtes effectuées dans sept écoles primaires rurales, l'étude débute par une évocation historique de la période 1954-1991. Contexte (objectifs, types de villages observés, structure éducative) et méthodologie de recherche sont décrits avant la présentation des résultats statistiques. Les performances sont liées à de multiples variables, mais l'amélioration du processus éducatif passe par une plus grande participation des familles et de la communauté villageoise.

ILON Lynn, MOOCK Peter, "School attributes, household characteristics, and demand for schooling: a case study of rural Peru", International Review of Education = Revue internationale de pédagogie, 1991, vol. 37, n 4, p. 429-451.

L'étude s'intéresse aux raisons pour lesquelles le taux de scolarisation est moins élevé, au Pérou, dans les zones rurales. Le coût financier de l'école joue un rôle important dans la décision des parents quant à l'inscription scolaire et à la poursuite d'études par leurs enfants.

VIGNER Gérard et al, "Scolarisation et monde rural : dossier ", Diagonales, février 1996, $n^{\circ} 37$, p. 14-35.

Un ensemble d'articles s'interroge sur les relations entre ces deux notions en Afrique. L'histoire de l'école rurale dans l'ex-Afrique occidentale française et le bilan de l'expérience de l'école Mandoul au Tchad illustrent les évolutions. L'adaptation nécessaire pour lier école et développement est parfois difficile, mais des solutions existent.

INDEX

Mots-clés : école rurale, milieu rural, politique éducative 